\title{
Cascade Simulations of Unidirectional Fiber Optical Parametric Oscillators
}

\author{
Ioannis Begleris, Peter Horak \\ Optoelectronics Research Centre, University of Southampton, Southampton, SO17 1BJ, UK \\ Email: i.begleris@soton.ac.uk
}

\begin{abstract}
The numerical model of a Fiber Optical Parametric Oscillator (FOPO) is presented. The model is simulated for varying losses within the fiber resonator and physical mechanisms that cause reduction in the efficiency of the device are identified.
\end{abstract}

\section{INTRODUCTION}

Four wave mixing (FWM) is a nonlinear optical processes exhibited in conventional silica fibers. This is the gain medium behind Fiber Optical Parametric Amplifiers (FOPA) where light of a certain pump wavelength propagating through a fiber is converted into two separate waves at different wavelengths labeled the signal and idler. The efficiency and bandwidth of this conversion are primarily determined by the fiber dispersion which in turn depends on the transverse refractive index profile of the fiber. However, along the fiber this profile can vary, resulting in a substantial decrease in the conversion efficiency. One way to limit this effect is to use only a short fiber length but re-circulate the signal in the fiber. This leads to coherent build-up of signal power in this fiber resonator which subsequently enhances the energy transfer from the pump to the idler. This system is termed a Fiber Optical Parametric Oscillator (FOPO) [1].

Recently FOPOs have attracted attention as systems for converting energy to wavelengths where no conventional laser sources exist. In particular the experimental work conducted in [2] demonstrated high conversion efficiency using a unidirectional FOPO with a combination of Wavelength Division Multiplexers (WDM) and a Highly Nonlinear Fiber (HNLF). In this article we present our model that simulates a unidirectional FOPO. By using this model we are able to visualize the build-up of optical power within the resonator on time scales too short for experimental observation, which gives new insights into efficiency decreasing factors.

\section{TheOreticAl AND NumericAl Method}

Figure 1 represents a schematic of the system. A CW pump wave is launched at position 0 into WDM1 and enters the resonator. The light field then passes through the HNLF where the signal and idler are created. Afterwards the field is inserted into WDM2 where the idler and the residual pump are extracted from the resonator while the signal is again combined with the pump within WDM1 for the next round trip. The numerical method is similar to the study conducted in [3], however here the system is modeled using frequency

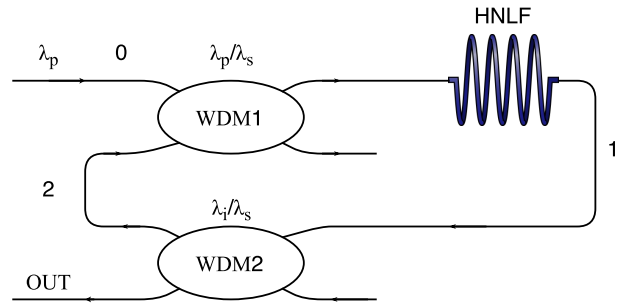

Fig. 1. Schematic diagram of the unidirectional FOPO apparatus.

dependent WDMs instead of a combination of a beam splitter and band pass filter.

The WDMs are modeled as $2 \times 2$ fiber couplers using the Scattering Matrix (SM) representation with two input and output ports. If spectra $U^{(i)}$ are inputted to ports $i=1,2$ then the outputs at ports $i=3,4$ are calculated using [4]:

$$
\left[\begin{array}{l}
U^{(3)} \\
U^{(4)}
\end{array}\right]=\left[\begin{array}{cc}
\sqrt{\epsilon(f)} & j \sqrt{1-\epsilon(f)} \\
j \sqrt{1-\epsilon(f)} & \sqrt{\epsilon(f)}
\end{array}\right]\left[\begin{array}{l}
U^{(1)} \\
U^{(2)}
\end{array}\right],
$$

where $\epsilon(f)$ is the frequency dependent ratio of power transferred from port 1 to port 3. $\epsilon(f)$ is approximated as a square of a sinusoidal function which has a maximum at the wavelength desired at port 3 and a minimum at port 4 . In particular, $\epsilon=1$ for WDM1 at the pump wavelength and $\epsilon=0$ at the signal wavelength. Equivalently the minima and maxima of WDM2 are situated at the idler and signal wavelength, respectively. For instances where there is no input to the ports random quantum noise is used.

Propagation within the HNLF is described by the Nonlinear Schrödinger Equation (NLSE) [1]:

$$
\begin{aligned}
& \frac{d A}{d z}=j \sum_{n \geq 2} \frac{\beta_{n}}{n !}\left(j \frac{\partial}{\partial t}\right)^{n} A(z, t)+j \gamma\left(1+\frac{j}{\omega_{p}} \frac{\partial}{\partial t}\right) \times \\
& \left(\left(1-f_{r}\right)|A(z, t)|^{2}+f_{r} \int h(\tau)|A(z, t-\tau)|^{2} d \tau\right) A(z, t),
\end{aligned}
$$

where $A(z, t)$ is the amplitude of the wave, $\omega_{p}$ the pump angular frequency, $\beta_{n}$ the $n_{t h}$ Taylor expansion coefficient of the dispersion at the pump frequency, $\gamma$ the nonlinear parameter, $f_{r}$ the fractional contribution of Raman scattering to the total nonlinearity and $h(\tau)$ the Raman response function. Equation (2) is solved at each resonator round trip using the split-step Fourier method and an adaptive step size RungeKutta method is used for the integration of the nonlinearity. 


\section{RESULTS}

For these particular simulations we use the photonic crystal fiber and the parameters provided in [3] where we assume that all parametric processes occur on a single polarization of light aligned to the slow fiber axis. The fiber is quoted to have $\gamma=10^{-2} \mathrm{~W} / \mathrm{m}$, a zero dispersion wavelength situated at $\lambda_{d}=1051.85 \mathrm{~nm}$ and the Taylor expansion coefficients at that wavelength are listed in [5].

The efficiency of conversion to the idler wavelength depends upon how much signal is oscillating within the resonator. Unlike experimental setups the numerical model provides the ability to visualize the signal buildup within the resonator. In order to highlight the importance of attenuation within the resonator a loss is added to the system at position 2 (see Fig. 1) of the FOPO. The loss is modeled using an SM representation, Eq. (1), where $\epsilon$ is considered constant in frequency.

As an illustration, $4.3 \mathrm{~W} \mathrm{CW}$ pump power situated at $1048.17 \mathrm{~nm}$ is launched into the system at position 0 . This results in the generation of a signal and an idler in the HNLF situated at $\lambda_{s} \approx 1.2 \mu \mathrm{m}$ and $\lambda_{i} \approx 0.93 \mu \mathrm{m}$, respectively. Fig. 2 depicts the power of the signal at position 1 as a function of round trips taken for an applied loss of $1.4 \mathrm{~dB}$ and $1.5 \mathrm{~dB}$. The power of the signal within the resonator initially increases for both losses, with the lower loss case leading to larger gain and thus more rapid power build up. However, this smooth increase of power is followed by a regime of rapid fluctuations. In the case of $1.5 \mathrm{~dB}$ loss, these take the form of damped periodic oscillations and the signal power approaches a stationary state on a time scale of order $10^{4}$ round trips. For the slightly smaller loss of $1.4 \mathrm{~dB}$, the average signal power is higher, but the fluctuations are irregular and show no convergence towards a stationary state on the time scale shown.

To gain further insight, we show the corresponding signal spectra within the FOPO in Fig. 3. Here we see that for $1.5 \mathrm{~dB}$ loss the signal spectrum converges towards a single smooth peak at the expected signal wavelength around 1.2 $\mu \mathrm{m}$. For $1.4 \mathrm{~dB}$ loss, on the other hand, the signal broadens considerably and a weak but noticeable cascaded FWM signal at $1.4 \mu \mathrm{m}$ is established despite the large losses per round trip experienced at this wavelength due to the WDMs. The spectral broadening and the appearance of this additional sideband indicate that above a certain power level nonlinear interactions occur between several wavelength bands beyond the simple system of pump, signal, and idler. The resulting complex dynamics can then lead to the instability of the observed signal power, shown in Fig. 2.

\section{CONCLUSION}

We presented a computational model of a unidirectional FOPO to study aspects of the system dynamics not normally accessible experimentally. As an example we investigated the initial dynamics after switching on and observed the transition from stable to unstable behavior for sufficiently high powers, which we attribute to the nonlinear coupling to cascaded FWM signals. For future studies our model is capable of simulating, e.g., a variety of fibers and pulsed FOPO operation, hence

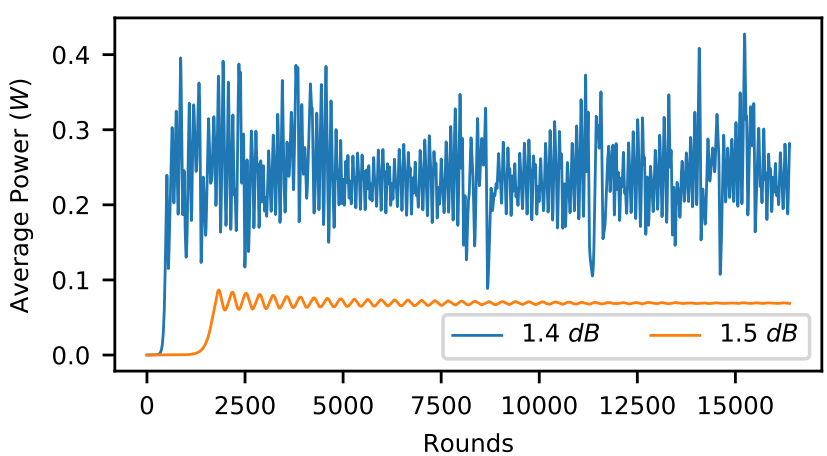

Fig. 2. Average power of the signal within the FOPO resonator as a function of number of round trips for two different losses per round trip at $\lambda_{s}$.

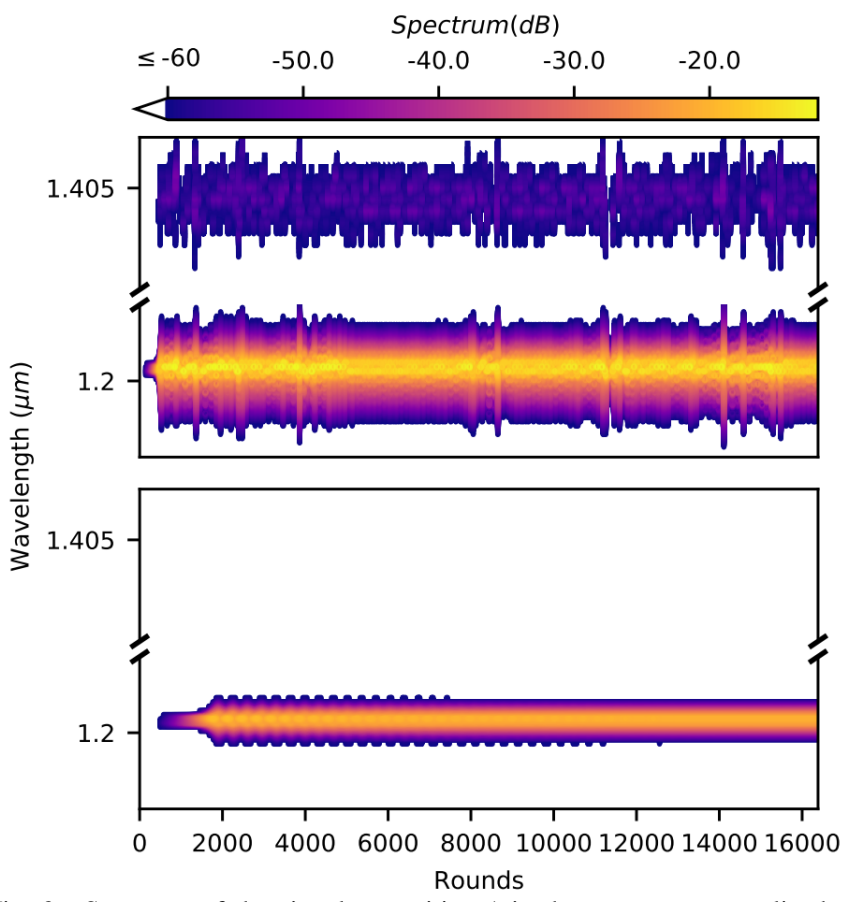

Fig. 3. Spectrum of the signal at position 1 in the resonator normalized to the input power. A loss of $1.4 \mathrm{~dB}$ (top) and $1.5 \mathrm{~dB}$ (bottom) is applied at position 2 .

providing a useful tool in optimizing FOPO systems for high conversion efficiency.

This work was supported by the EPSRC CDT in Next Generation Computational Modelling (grant EP/L015382/1).

\section{REFERENCES}

[1] G. P. Agrawal, Nonlinear Fiber Optics, 5th ed. Academic Press, 2001.

[2] E. A. Zlobina, S. I. Kablukov, and S. A. Babin, "High-efficiency CW Allfiber Parametric Oscillator Tunable in 0.92-1 $\mu$ m Range," Optics Express, vol. 23, p. 833, 2015.

[3] W. Q. Zhang, J. E. Sharping, R. T. White, T. M. Monro, and S. Afshar V, "Design and Optimization of Fiber Optical Parametric Oscillators for Femtosecond Pulse Generation." Optics Express, vol. 18, pp. 17294-305, 2010.

[4] G. Keiser, Optical Fiber Communications. McGraw-Hill, 1983.

[5] E. A. Zlobina, S. I. Kablukov, and S. A. Babin, "Phase matching for parametric generation in polarization maintaining photonic crystal fiber pumped by tunable yb-doped fiber laser," J. Opt. Soc. Am. B, vol. 29, no. 8, pp. 1959-1967, Aug 2012. 